\title{
A NEW FORM OF THE GENERALIZED CONTINUUM HYPOTHESIS
}

\author{
BY HERMAN RUBIN
}

Communicated by Paul R. Halmos, June 2, 1959

We shall prove that the following condition is equivalent to the generalized continuum hypothesis:

(*) For all transfinite cardinals $p$ and $q$, if $p$ covers $q$, then for some $r$, $p=2^{r}$.

By $p$ covers $q$, we mean that $p>q$ and for no $r$ is $p>r>q$.

The generalized continuum hypothesis is usually stated in the form that, for any transfinite cardinal $p, 2^{p}$ covers $p$. We shall use instead the equivalent form $[2 ; 4]$ as the logical product of the aleph hypothesis $2 \boldsymbol{\aleph}_{\alpha}=\boldsymbol{\aleph}_{\alpha+1}$ and the axiom of choice.

If the generalized continuum hypothesis holds, then $\left(^{*}\right)$ follows easily. For then if $p$ and $q$ are transfinite and $p$ covers $q$, then by the axiom of choice for some $\alpha, q=\boldsymbol{\aleph}_{\alpha}$ and $p=\boldsymbol{\aleph}_{\alpha+1}$ and so by hypothesis $p=2^{q}$.

Let us now proceed to the converse. First we shall prove the aleph hypothesis. Since for all $\alpha, \boldsymbol{\aleph}_{\alpha+1}$ covers $\boldsymbol{\aleph}_{\alpha}$, we have $\boldsymbol{\aleph}_{\alpha+1}=2^{r}$ for some $r$. Since $r<2^{r}, r$ must be $\boldsymbol{\aleph}_{\gamma}$ for some $\gamma$. Let $\beta(\alpha)$ be the smallest such $\gamma$. We clearly have $\beta(\alpha)<\alpha+1$. However, $\beta(\alpha)$ is a strictly monotone function of $\alpha$ and hence is greater than or equal to $\alpha$. Thus $\beta(\alpha)=\alpha$ and the aleph hypothesis is proved.

Let us now demonstrate that the axiom of choice follows from (*). We first prove from the axioms of set theory the following

Lemma. ${ }^{1}$ If $2^{p} \leqq q+\aleph_{\alpha}$, where $p$ and $q$ are transfinite, then $p<q$ or $p<\boldsymbol{\aleph}_{\alpha}$.

For since $p<2^{p}, p=s+t$, where $s \leqq q$ and $t \leqq \boldsymbol{\aleph}_{\alpha}$. Then $2^{p}=2^{\bullet} 2^{t}$, and by [2] either $2^{s} \leqq \boldsymbol{N}_{\alpha}$ or $2^{t} \leqq q$. But in the first case $s+t \leqq \boldsymbol{N}_{\alpha}$ since both $s$ and $t$ are, and in the second case $s+t \leqq q$ since both $s$ and $t$ are, and in addition $t$ is less than or equal to an aleph. Thus we have demonstrated the lemma except for the strictness of the inequalities. That follows since $[2 ; 5]$ if $2^{p} \leqq p+r$, then $2^{p} \leqq r$, and $p<r$, q.e.d.

For any transfinite cardinal $p$, let us denote by $p^{*}$ the smallest aleph [1] not less than or equal to $p$. Tarski [3] has shown that if $p$ is transfinite then $p+p^{*}$ covers $p$. But since by [2] the mapping $p \rightarrow p^{*}$

${ }^{1}$ This lemma is due to Professor A. Tarski and is an extension of the author's original argument. 
preserves addition and since $\boldsymbol{\aleph}_{\alpha}^{*}=\boldsymbol{\aleph}_{\alpha+1}$, it follows that if $\boldsymbol{\aleph}_{\alpha+1}$ is not less than or equal to $p$ then $p+\boldsymbol{\aleph}_{\alpha+1}$ covers $p+\boldsymbol{\aleph}_{\alpha}$. Then by $(*)$, $p+\boldsymbol{\aleph}_{\alpha+1}=2^{q}$, and we have $q<p$ or $q<\boldsymbol{N}_{\alpha+1}$. However, if we choose $\aleph_{\alpha+1} \geqq\left(2^{p}\right)^{*}$, the first alternative is impossible, and $q$ is an aleph. Then by the aleph hypothesis, $2^{q}$ is an aleph, and so $p$ is an aleph.

\section{BIBLIOGRAPHY}

1. F. Hartogs, Über das Problem der Wohlordnung, Math. Ann. vol. 76 (1915) pp. 438-443.

2. A. Lindenbaum and A. Tarski, Communications sur les recherches de la theorie des ensembles, C.R. Soc. Sci. Lett. Varsovie, Classe III vol. 19 (1926) pp. 299-330.

3. A. Tarski, Theorems on the existence of successors of cardinals, and the axiom of choice, Nederl. Akad. Wetensch. Proc. Ser. A vol. 57 (1954) pp. 26-32.

4. W. Sierpinski, L'hypothèse généralisée du continu et l'axiome du choix, Fund. Math. vol. 34 (1947) pp. 1-5.

5. W. Sierpinski, Demonstration de l'égalite $2^{m}-m=2^{m}$ pour les nombres cardinaux transfinis, Fund. Math. vol. 34 (1947) pp. 113-118.

UNIVERSITY OF OREGON 